\title{
High Quality Electron Beams from Laser Wakefield Acceleration: A New Compact Source for Electron Microscopes?
}

\author{
John Mansfield*, John Nees**, Gerard Mourou*** and Almantas Galvanauskas**** \\ *University of Michigan North Campus Electron Microbeam Analysis Laboratory, 417 SRB, 2455 \\ Hayward, Ann Arbor, MI 48109-2143, USA \\ **Center for Ultrafast Optical Science, 1012 Gerstacker Building, Ann Arbor, MI 48109-2099, USA \\ *** Laboratoire d'Optique Appliquee, ENSTA - Ecole Polytechnique, Chemin de la Huniere, 91761 \\ Palaise au Cedex, France \\ **** Center for Ultrafast Optical Science, 6112 ERB I, Ann Arbor, MI 48109-2099, USA
}

The relativistic and non-linear properties of ultra-fast, very high intensity beams of light present some enticing new possibilities for the structural characterization of materials, not only at the nanometer length scale, but also at the sub-nanosecond, picosecond, and even attosecond, time scale. High-resolution transmission electron microscopy (TEM) has now reached into the picometer regime[1-3] and, after many years of limited advance in the time domain, Dömer and Bostanjoglo4 have reported TEM and electron diffraction observations in the nanosecond time regime. They used the pulsed laser photocathode, developed by Williamson and Mourou [5] and used successfully by others [6],[7] in a 1960s Siemens 1A TEM.

Although the time resolution experiments require extra instrumentation to be added to the standard electron column of the microscope, i.e. the photocathode system and the ultrafast camera systems, that would apparently increase the complexity of the whole microscope system, there is now evidence that the electron source and accelerator of the standard TEM could be replaced by a compact table system [8]-[10]. It is possible that such a compact source could significantly lower the overall cost of the microscope. The compact system would rely upon a laser accelerator, in which electrons are accelerated by the electric field of a plasma wave (the wakefield) driven by a very-short (a few fs) high-intensity $(\sim \mathrm{mJ})$ focused laser pulse, a concept first described by Modena et al in 1995 [11] and later demonstrated experimentally [12],[13]. Electrons have been shown to be accelerated very efficiently in this process, from rest to $\sim 100 \mathrm{MeV}$ in a few millimeters, a distance considerably shorter than in a conventional accelerator or in the gun of a conventional electron microscope, where the ultimate voltage is typically only in the few hundred $\mathrm{KeV}$ range. In 2002, Malka et al [14] showed that well collimated beams of $10^{8}$ electrons could be produced by a laserdriven wakefield. However, the energy spread of the beams was very large $(\sim 100 \%)$ because the electrons we trapped and accelerated in the high amplitude plasma wave. Injection of tight bunches of electrons near the peak of the plasma wave, to keep them all at approximately the same energy, was expected to be difficult, since the wavelength of the plasma wave is very short (a few $\mu \mathrm{m}$ ). However, in late 2004, three groups reported that they had found a new regime where electrons are injected into a narrow region of space and are picked up by the plasma wave as a single group and "surf" the wave together at a similar energy [12]-[14]. The energy spread of the resultant beams is still very large by TEM standards, Faure et al [12] note that their beams were $170 \pm 20 \mathrm{MeV}$, but the possibilities for a TEM electron source are very intriguing.

There are, of course, a number of problems to overcome. These include: the number density of the electron pulse, the repetition rate of the source, the size of the source and the interfacing of the source with the electron optical column. Current wakefield excited sources use plasma jets, which would prove to be a problem in a TEM system. Although it may not seem that there are very many 
electrons in these electron pulses $\left(10^{8-9}\right)$, the fact that they are in a very short duration pulse (a few fs) means that passing them through the focus points in the column an electron microscope would lead to space charge problems as they would interact with one another. However, since it would be necessary to use a monochromator to improve the energy spread of the beam and this would reduce the number density of the pulse.

The repetition rate of the electron pulse will be dictated by the ultrafast laser and the current lasers that can generate the high intensities that are necessary for wakefield acceleration operate at comparatively low repetition rate $(<10 \mathrm{~Hz})$. For the source to replace a conventional TEM source it would need to appear to be "continuous wave" and have a repetition rate of the order of $30 \mathrm{~Hz}$ or more. If it were also to be used for time resolved studies, it would need to be in at least the kilohertz or megahertz range. Additionally, current ultrafast laser systems tend to occupy laboratories of the same approximate scale as a TEM lab and one would question how using such a laser as a source would make the TEM more compact! Recent advances in the development of fiber lasers promises to address the issues of both repetition rate and compactness. Galvanauskas et al have demonstrated a milli-Joule short pulse all-fiber laser and Limpert et al have demonstrated a high average power fiber laser [15],[16]. The final dimensions of the high average power, high repetition rate fiber lasers are expected to be similar to that of the case of the average personal computer and it is not unreasonable to assume that the full accelerator system would be only two or three times that size. A true desk-top high-voltage electron source is, therefore, an intriguing and attractive possibility.

References

1 Nellist, P. D. and S. J. Pennycook (1998). Physical Review Letters 81: 4156-59.

2 O'Keefe, M. A., C. J. D. Hetherington, et al. (2001). Ultramicroscopy 89(4): 215-241.

3 O'Keefe, M. A., E. C. Nelson, et al. (2001). "Sub-Ångstrom resolution of atomistic structures below 0.8Å." Philosophical Magazine B 81(11): 1861-1878.

4 Dömer, H. and O. Bostanjoglo (2003). Review of Scientific Insruments 74(10): 4369-4372.

5 Williamson, S. and G. Mourou (1982). Applied Physics B-Photophysics and Laser Chemistry 28(2-3): 249-250.

6 Elsayed-Ali, H. E. and G. A. Mourou (1988). App. Phys. Lett. 52: 103-104.

7 Cao, J., Z. Hao, et al. (2003). Applied Physics Letters 83: 1044.

8 Faure, J. Glinec, et al. (2004). Nature 431:

9 Mangles, S.P.D., Murphy, C.D. et al (2004). Nature 431: 535,538.

10 Geddes, C.G.R., Toth, Cs et al. (2004). Nature 431: 538,541.

11 Modena, A., Z. Najmudin, et al. (1995). Nature 337: 606.

12 Umstadter, D., S. Y. Chen, et al. (1996). Science 273: 472-475.

13 Umstadter, D., J. K. Kim, et al. (1996). Physical Review Letters 76: 2073-2076.

14 Malka, V. et al (2002). Science 298: 1596,1600

15 Galvanauskas, A., Z. Sartania, et al. (2001). Conference on Lasers and Electro-Optics (CLEO 2001) Baltimore, MD, May 6 - 11, 2001, invited paper CMA1.

16 Limpert, J. and e. al (2003). paper WE3 in Advanced Solid State Photonics 2003. 